(2) Open Access Full Text Article

\title{
A randomized, placebo-controlled, single-blinded, split-faced clinical trial evaluating the efficacy and safety of KLOX-00 I gel formulation with KLOX light-emitting diode light on facial rejuvenation
}

\author{
This article was published in the following Dove Press journal: \\ Clinical, Cosmetic and Investigational Dermatology \\ 13 May 2016 \\ Number of times this article has been viewed
}

\section{Andreas Nikolis' \\ Steven Bernstein ${ }^{2}$ \\ Brian Kinney ${ }^{3}$ \\ Nicolo Scuderi ${ }^{4}$ \\ Shipra Rastogi ${ }^{5}$ \\ John S Sampalis ${ }^{6}$}

'Victoria Park, Plastic Surgery Section, Westmount, QC, Canada;

${ }^{2}$ Dermatology Department, University of Montreal Health Centre, Montreal, QC, Canada; ${ }^{3}$ Department of Plastic Surgery, USC School of Medicine, Beverley Hills, CA, USA; ${ }^{4}$ Department of Plastic and Reconstructive Surgery, La Sapienza, Rome, Italy; ${ }^{5} \mathrm{KLOX}$ Technologies, Laval, ${ }^{6}$ SS Medical Research, Montreal, QC, Canada
Correspondence: Andreas Nikolis Victoria Park, Plastic Surgery Section, 376 Victoria, Suite 400, Westmount, Quebec, H3ZIC3 Canada

Tel + I 5 I4 4887722

$\mathrm{Fax}+\mathrm{I} 5144883830$

Email anikolis@vicpark.com
Purpose: Many treatment modalities exist to counteract the effects of cutaneous aging. Ablative methods have been the mainstay for nonsurgical facial rejuvenation. In recent years, nonablative techniques have been developed with the aim of achieving facial rejuvenation without epidermal damage. Light-emitting diode (LED) photorejuvenation is a novel nonablative technique that induces collagen synthesis through biophotomodulatory pathways.

Materials and methods: A single-center, randomized, single-blinded, placebo-controlled, split-faced clinical trial was designed. Thirty-two patients were enrolled for a 12-week study. Patients were randomized into one of four groups: Group A, treatment with KLOX-001 gel formulation and white LED (placebo) light; Group B, treatment with a placebo/base gel (no active chromophore) formulation and KLOX LED light; Group C, treatment with KLOX-001 gel formulation and KLOX LED light; and Group D, treatment with the standard skin rejuvenating treatment $(0.1 \%$ retinol-based cream). Patients received treatment at weeks $0,1,2$, and 3, and returned to the clinic at weeks 4,8 , and 12 for clinical assessments performed by an independent, blinded committee of physicians using subjective clinician assessment scales. Tolerability, adverse outcomes, and patient satisfaction were also assessed.

Results: Analysis demonstrated that the KLOX LED light with KLOX placebo/base gel and the KLOX LED light + KLOX-001 gel formulation groups were superior to standard of care and KLOX-001 gel formulation with placebo light on subjective clinical assessment and multiple wrinkle scales, with statistically significant results obtained for brow positioning, perioral wrinkling, and total wrinkle score.

Conclusion: The study results show that KLOX LED light with KLOX-001 gel formulation and KLOX LED light with KLOX placebo/base gel are effective, safe, well-tolerated, and painless treatment modalities for skin rejuvenation.

Keywords: biophotonics, skin rejuvenation, chromophore, rejuvenation, non-invasive

\section{Introduction}

Cutaneous aging is a natural inevitable process leading to a considerable desire for many patients to achieve a more youthful look. Many treatment modalities exist, both medical and nonmedical, to improve an individual's physical appearance as well as their perception of their appearance, consequently influencing psychological well-being. ${ }^{1}$ Cutaneous aging results from a combination of intrinsic biological factors and extrinsic environmental factors ${ }^{2}$ leading to a variety of clinical manifestations, including fine lines and rhytides, dyspigmentation, telangiectasia, elastosis, and textural irregularities. ${ }^{3}$ 
Histologically, aged skin presents with epidermal thickening, diminished collagen synthesis leading to dermal atrophy and loss of dermal papillae, as well as a fragmented and disorganized elastin network. ${ }^{3-8}$ Extrinsic factors are many and include tobacco use, sleeping positions, repetitive facial expressions, and ultraviolet radiation from the sun. ${ }^{1,3}$ Current nonsurgical therapies are centered on ablative methods of skin rejuvenation. These include chemical peels, dermabrasion, and laser resurfacing. ${ }^{9-13}$ Ablative methods remove the epidermis and induce a controlled form of wounding, thus promoting collagen synthesis and dermal extracellular matrix remodeling. ${ }^{13-15}$ These procedures may require complex postoperative care, can be quite painful, and can also lead to significant complications. ${ }^{9,16,17}$

The development of nonablative techniques stems from patients' desire to decrease discomfort and downtime and clinicians' desire to offer simpler, effective alternatives with fewer side effects. ${ }^{13,18-21}$ Nonablative skin rejuvenation can be classified into two types: type I photorejuvenation targets primarily telangiectasia and irregular pigmentation, whereas type II photorejuvenation aims for wrinkle and fine line reduction and skin tightening. ${ }^{13,22}$

Light-emitting diode (LED) photorejuvenation is a novel noninvasive procedure that is nonthermal, atraumatic, and induces collagen synthesis through biophotomodulatory pathways. ${ }^{9}$ Subtypes of LED photomodulation include, but are not limited to, the photodynamic and the biophotonic platforms. The biophotonic platform is distinct from the photodynamic one in that both use a combination of LED light and a chromophore-rich gel; however, in the biophotonic platform, chromophores act topically to enhance the effects of the LED light and are neither absorbed nor metabolized. Many clinical trials have shown the efficacy of LED therapy in skin rejuvenation. ${ }^{9,20,23-29}$ They include increased collagen deposition and decreased collagen degradation by upregulation of fibroblast activity. ${ }^{9,19}$

Preliminary studies with the KLOX-001 formulation demonstrate in vitro antibacterial efficacy and a stimulatory effect on human dermal fibroblasts. Additionally, KLOX-001 gel formulation and KLOX LED light biophotonic combination has been shown to decrease necrosis in the rat flap model, as well as increase collagen fibrin deposition. ${ }^{30}$ The KLOX-001 topical gel formulation comprises principally of an oxidant, chromophores, and a hydrophilic gel carrier. The KLOX LED light delivers symmetrical peak wavelengths in the visible range $(400-470 \mathrm{~nm})$ with a peak at $446 \mathrm{~nm}$. It produces a power density of $150 \mathrm{~mW} / \mathrm{cm}^{2}$ at a distance of $5 \mathrm{~cm}$ from the light source, which meets the US Food and
Drug Administration and the American National Standard Institute Z135 regulations on optical exposure to skin. As the blue light illuminates the chromophores, it gets converted into a broad range of wavelengths.

This study was designed to assess the efficacy, tolerability, and safety of KLOX-001 gel formulation with KLOX LED light on skin rejuvenation in women. The primary endpoint was subjective clinical assessment of KLOX-001 gel formulation and KLOX LED light, alone or in combination, on skin rejuvenation of the face after four weekly treatments. Secondary endpoints included the effects of treatment with KLOX-001 gel formulation and KLOX LED light on the severity of glabellar lines, marionette lines and perioral wrinkling, nasolabial wrinkle severity, forehead wrinkling, periocular wrinkling and crow's feet, cheek wrinkling, overall photo-damage, subjective patient satisfaction with treatment, pain, and erythema, histologic changes, as well as patient safety and tolerability.

\section{Materials and methods}

A single-center, randomized, single-blinded, placebocontrolled, split-control-faced clinical trial was designed. The KLOX internal clinical number for this study is CL-K1001001. The duration of active phase of the study was 12 weeks. Ethics approval was obtained from the Canadian SHIELD Ethics Review Board, which reviewed the study protocol and found it to be acceptable. Written consent was also obtained from the patients by KLOX Technologies for the use of the photos in this paper.

All eligible patients (Table 1) who agreed to participate in the study and signed an informed consent form were randomized in a 1:1:1:1 ratio into one of the following four groups: Group A received treatment for half the face with KLOX-001 gel formulation and white LED (placebo) light and the contralateral side was treated with a placebo (base gel - no chromophores) formulation and light; Group B received treatment for half the face with a placebo/base gel formulation and KLOX LED light and the contralateral side was treated as Group A; Group C received treatment for half the face with KLOX-001 gel formulation and KLOX LED light and the contralateral side was treated as the other controls; and Group D received treatment for half the face with the standard skin rejuvenating treatment $(0.1 \%$ retinol-based cream) and the control side received a basic moisturizer. After initial randomization, patients were randomly allocated in a 1:1 ratio to treatment of either right or left side.

Prior to treatment, all patients received a single microdermabrasion treatment of the full face at a maximum of 
Table I Study inclusion and exclusion criteria

\begin{tabular}{|c|c|}
\hline \multicolumn{2}{|c|}{ Inclusion criteria } \\
\hline I. & Female \\
\hline 2. & $30-65$ years of age \\
\hline 3. & Caucasian \\
\hline 4. & Fitzpatrick skin type $>$ Class II \\
\hline 5. & Willingness to undergo two biopsies in the retroauricular region \\
\hline 6. & Willingness to return for postoperative visits \\
\hline \multicolumn{2}{|c|}{ Exclusion criteria } \\
\hline I. & Facial cosmetic procedure in the last 6 months \\
\hline 2. & Neuromodulator treatments within last 6 months \\
\hline 3. & Injectable fillers within last 12 months \\
\hline 4. & Poly-L-lactic acid injections within last 3 years \\
\hline 5. & Laser therapy within last 6 months \\
\hline 6. & $\begin{array}{l}\text { Physical or psychiatric condition preventing the patient from } \\
\text { completing the study }\end{array}$ \\
\hline 7. & Use of medications that would predispose to bleeding/bruising \\
\hline 8. & Use of drugs known to increase photosensitivity \\
\hline 9. & Isotretinoin use within last 12 months \\
\hline 10. & Cortisone use within last 6 months \\
\hline II. & Pregnant or breast-feeding \\
\hline 12. & Marked facial asymmetry \\
\hline 13. & Active infection \\
\hline 14. & History of hypertrophic scarring \\
\hline 15. & History of radiation therapy to head or neck \\
\hline 16. & Dermatological comorbid disease \\
\hline 17. & Immunosuppression \\
\hline 18. & History of neuromuscular disorder \\
\hline 19. & Concurrent enrollment in similar study \\
\hline 20. & Prior surgery altering subcutaneous anatomy in the treated areas \\
\hline
\end{tabular}

48 hours from the beginning of the trial. Skin biopsies were taken from the retroauricular region at weeks 0 and 12 for a direct comparison of the effects of the treatment while minimizing confounding factors. During the active treatment period, there were a total of four weekly visits. Patients received treatment on one half of the face and the biopsy region. Groups A and C were treated with a $2 \mathrm{~mm}$ thick layer of KLOX-001 gel formulation on the experimental side and a nonchromophore placebo/base gel formulation on the control side. Groups B and C were treated with KLOX LED light by the light source at a distance of $5 \mathrm{~cm}$ from the skin surface (power density of $150 \mathrm{~mW} / \mathrm{cm}^{2}$ ) for a duration of 5 minutes per facial subunit to ensure complete facial coverage on the experimental side and with sham placebo light (white LED) on the control side. Group D received a standard skin rejuvenating treatment $(0.1 \%$ retinol-based cream) on the experimental side and a basic moisturizer on the control side.

During treatment, all patients were blinded with external eyelid protectors to shield the retina from direct illumination. Only the treatment segment of the face was exposed to light. A subjective patient assessment questionnaire was completed and a standardized clinical assessment was performed. Patients were asked to complete a short questionnaire for assessing their degree of satisfaction following each visit.

The primary endpoint of the study was the change in the clinical index from baseline (week 0) to week 12 following treatment, as determined by an independent, blinded committee of three physicians using a subjective 5-point scale from 0 to 4 ( $0=$ worse; $1=$ little or no improvement [0\%-25\%]; $2=$ some improvement $[26 \%-50 \%] ; 3=\operatorname{good}$ improvement [51\%-75\%]; and $4=$ excellent improvement [76\%-100\%]). In addition, the committee of physicians assessed: 1) severity of glabellar lines using the 4-point score for glabellar frown lines; $;^{31}$ 2) perioral and periorbital wrinkling using Fitzpatrick's 9-point wrinkle scoring system $^{32}$ (perioral wrinkling was also assessed using the Validated Grading Scale for marionette lines ${ }^{33}$ and the Validated Lip Fullness Grading Scale);34 3) nasolabial wrinkling using the modified Fitzpatrick Wrinkle Scale ${ }^{35}$ 4) forehead wrinkling using the 5-point photonumeric Forehead Lines Grading Scale; ${ }^{36} 5$ ) periocular wrinkling using the 5-point photonumeric Crow's Feet Grading Scale ${ }^{37} 6$ ) cheek wrinkling using Lemperle's 6-point Wrinkle Assessment Scale, Day's Wrinkle Severity Rating Scale, and Fitzpatrick's wrinkle scoring system; ${ }^{32,38,39} 7$ ) overall photo-damage using Glogau's classification of patient photoaging types and Fitzpatrick's wrinkle scoring system; ${ }^{32,40}$ and 8) patient's subjective satisfaction with treatment, as measured by the 5-point patient assessment index. The committee consisted of board-certified physicians who were trained in scoring using standardized patient photographs. They were not blinded to time elapsed from treatment, but were blinded to treatment type and side.

Thirty-two patients were enrolled. Sample size was calculated on the basis of detecting a $20 \%$ difference in primary outcome measure with a power of $80 \%$. The secondary outcome measure was the change in the total wrinkle score (TWS) from baseline to 4, 8, and 12 weeks. The TWS was calculated as the sum of patient's self-assessment of the horizontal, marionette, glabellar, and crow's feet wrinkle severity classified as $1=$ none, $2=$ mild, $3=$ moderate, and 5 $=$ severe. The TWS had a minimum value of 4 and maximum value of 20, with higher values indicating higher severity. Tolerability of treatment was assessed using the 4-point Pain Assessment Scale ( 1 = no pain; 2 = some pain; 3 = very painful, but bearable; and $4=$ unbearable pain). Descriptive statistics (including mean, standard deviation, median, range, and $95 \%$ confidence intervals) were produced for continuous 
scale variables and frequency distributions for categorical scale variables.

The chi-square statistic was used to assess the betweengroup differences with respect to the primary outcome measure. For the secondary outcome measures, the statistical analysis involved the use of multivariate general linear models, adjusting for patient's age and baseline values of the TWS. The general linear models were used to produce estimates of placebo-adjusted least-square mean estimates for the change in TWS at each visit. This was done by estimating the placebo-adjusted estimate of the TWS at each visit for each patient using a predictive linear regression model to offset the TWS of each KLOX-treated patient by the estimated standard of care (SOC) value. When compared to group-based adjustments, this method allows for less-biased placebo adjustment since each patient's TWS was offset by the estimated value that the individual patient would have achieved if she was treated by SOC. Tukey's Least Significant Difference test was used to estimate the pair-wise statistical significance while adjusting for multiplicity due to the six different pair-wise comparisons for each outcome every week.

A skin biopsy behind one ear was performed at weeks 0 and 12 on all subjects and stained with Hematoxylin, Phloxine, and Eosin, Gomori Trichrome, and Luna's methods according to standard protocols. A thorough histopathological examination of the sections at weeks 0 and 12 was performed by an experienced pathologist blinded to the treatment assignment.

Treatment-emergent adverse events (AEs) were summarized within each treatment group and presented according to the severity and the relationship to study treatment (probably related, possibly related, not related).

\section{Results}

Thirty-two patients satisfied the inclusion/exclusion criteria. Only two $(6.2 \%)$ subjects discontinued the study prior to completion; they were only lost to follow-up at week 12 (Table 2).

Analysis of subjective physician assessment demonstrated that the group treated with KLOX LED light + KLOX-001 gel formulation had superior performance when compared to the other treatment groups, with statistically significant results seen for changes in brow positioning $(P=0.001)$ and perioral wrinkling $(P=0.018)$ (Table 3, Figures $1-4)$.

Analysis of TWS least-square mean adjusted predicted value changes demonstrated that while the KLOX LED light with base KLOX-001 gel group was superior in the majority of the comparisons, the KLOX LED light + KLOX-001 gel group was also superior to SOC and KLOX-001 gel alone. This is seen across all treatment weeks (Figure 5) with statistically significant $P$-values for regression slopes when subjected to Student's t-test against the null hypothesis (Figure 6). While interpreting TWS results, higher values correlate to a less-favorable esthetic result, as they constitute a sum of four different scales.

Due to the small sample size, no formal statistical analyses were performed on the biopsy results. The average score and $\%$ of change from baseline with the Gomori Trichrome for each group was calculated, as it was the only parameter that did stand out clearly indicating an increase in the collagen from baseline to week 12 that was particularly notable ( $400 \%$ increase) in the group treated with the combination of KLOX-001 gel formulation and KLOX LED light (Table 4, Figure 7).

The most commonly reported treatment-related AEs were brow edema (13\%), erythema (19\%), and eyelid edema $(9 \%)$

Table 2 Summary of subject disposition during the study period

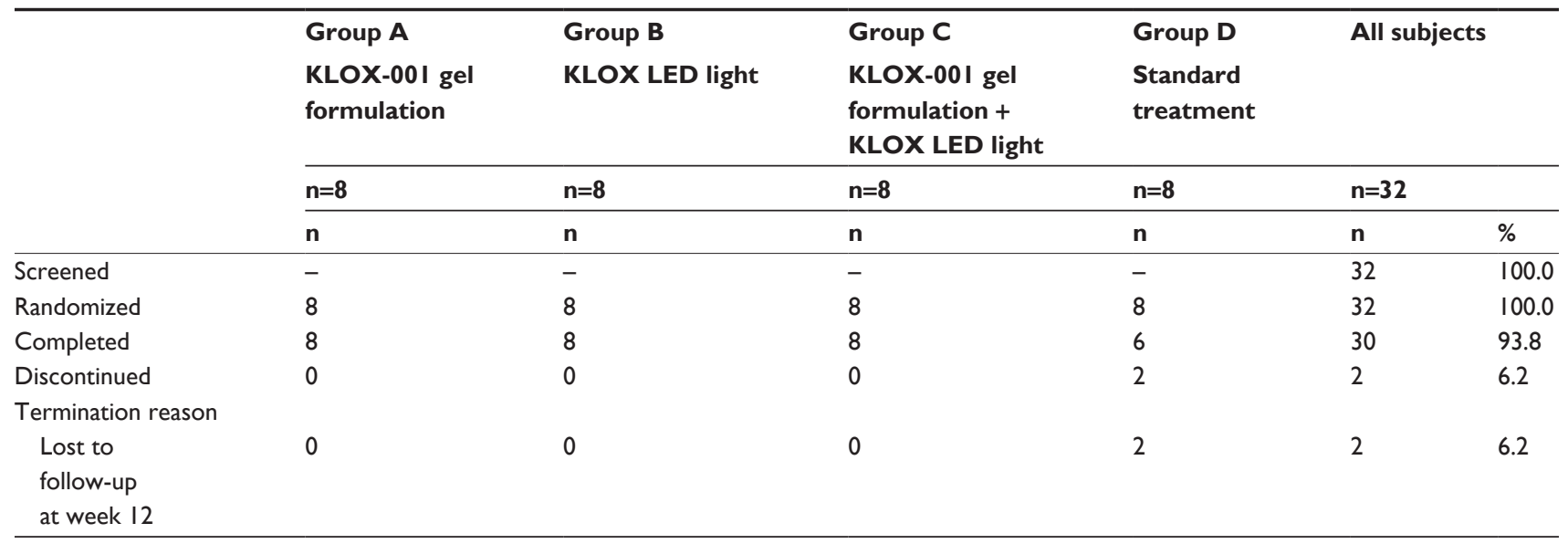

Abbreviation: LED, light-emitting diode. 


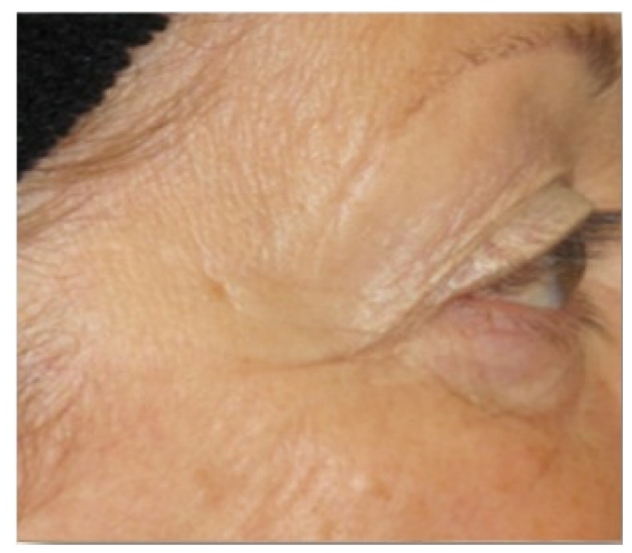

Week 0

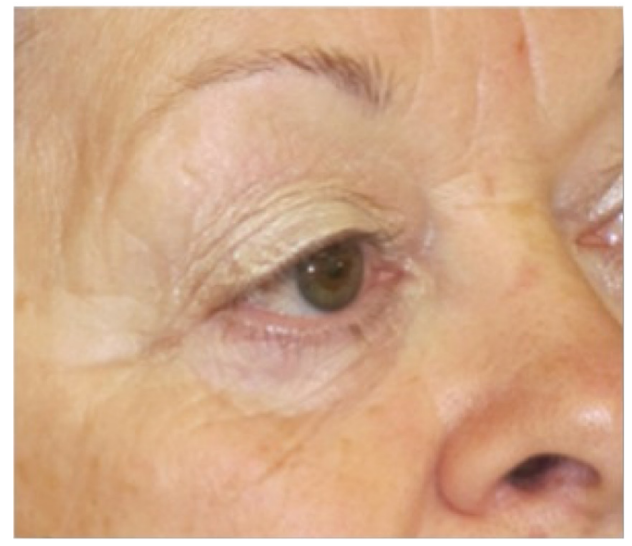

Week 0

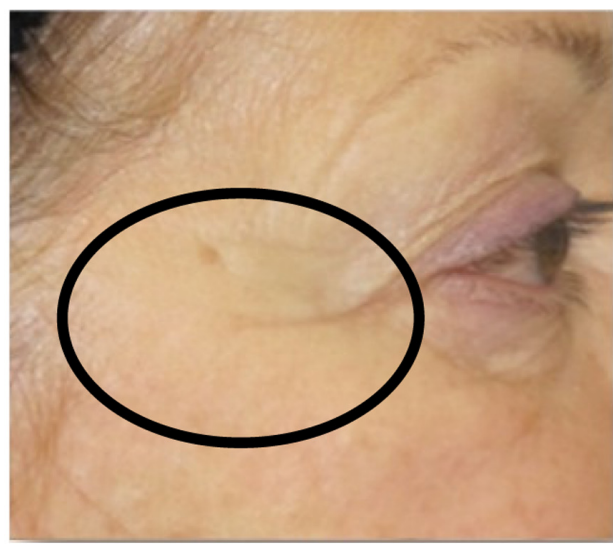

Week 12

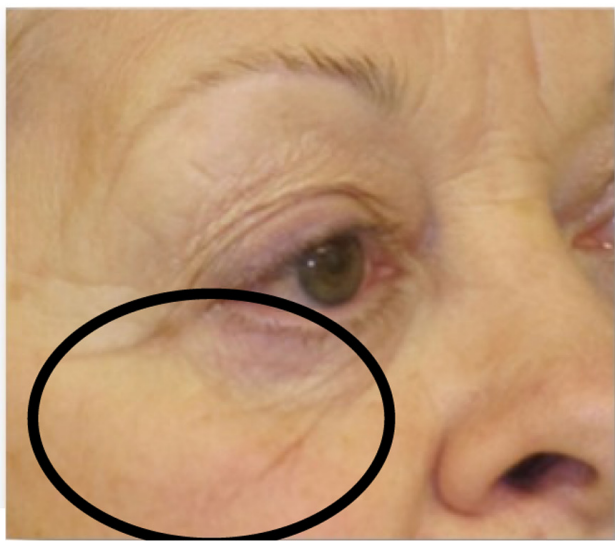

Week 12

Figure I Improvement of crow's feet and decrease in fine rhytides in the identified regions.

(Table 5). All the AEs reported were transient and mild in intensity, without any subject being discontinued from the study due to AE. No serious AEs or deaths were reported during the course of the study. The number of subjects reporting AEs was small and no clear differences were noted between the groups.

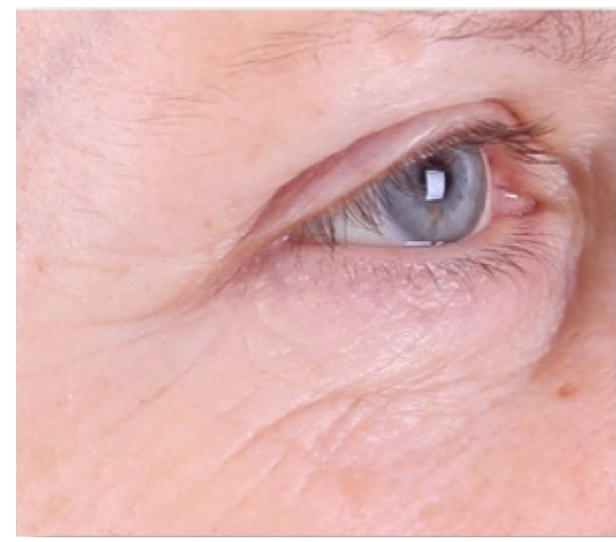

Week 0

\section{Discussion}

LED photorejuvenation presents with several advantages when compared to ablative and nonablative thermal methods. It is rarely painful and offers less downtime. The effects are thought to occur primarily through photomodulation of fibroblast, collagen deposition, as well as metalloprotease pathways.

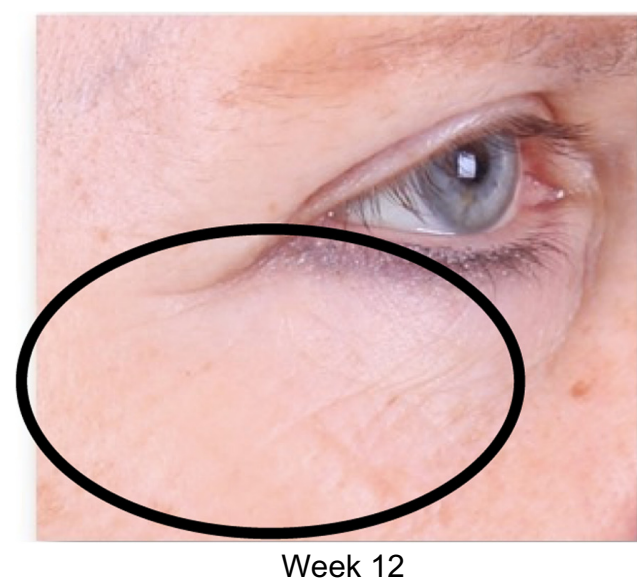

Figure 2 Improvement of crow's feet and decrease in fine rhytides in the identified lower lid region. 


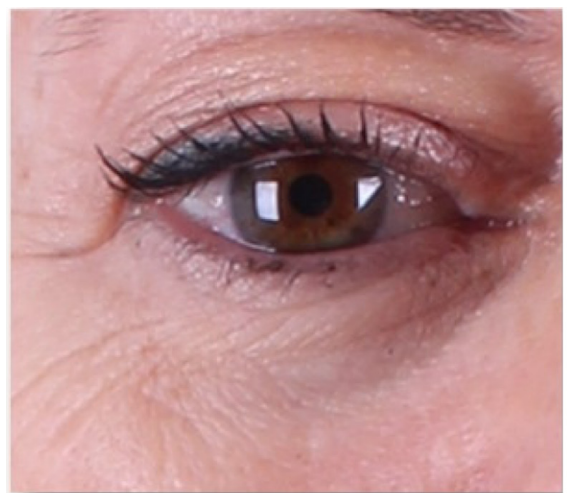

Week 0

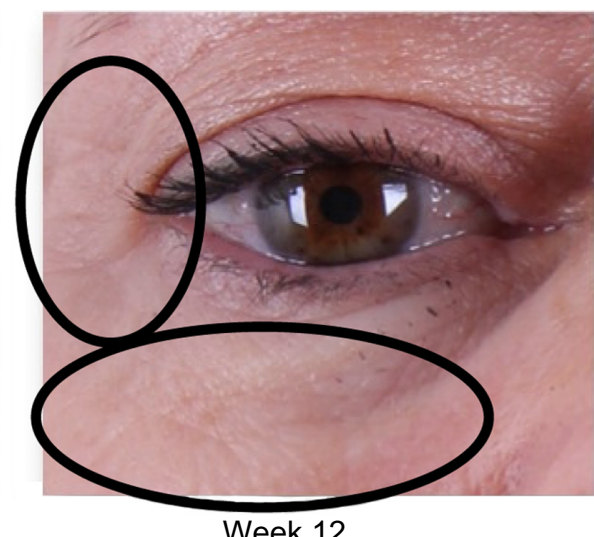

Week 12

Figure 3 Improvement of crow's feet and decrease in fine rhytides in the identified regions.

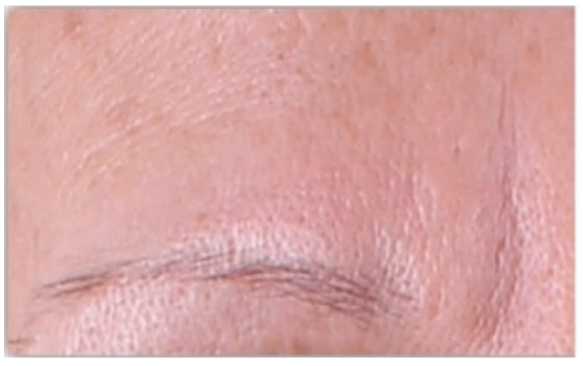

Week 0

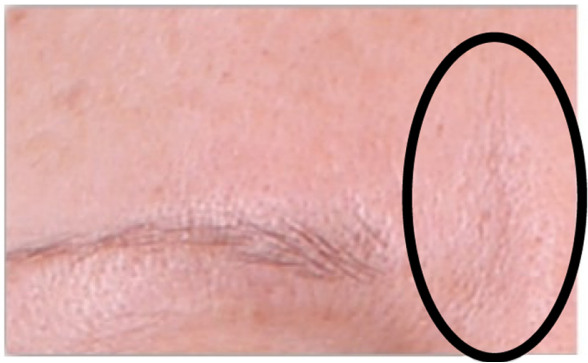

Week 12

Figure 4 Glabella improvement at week 12 (anterior view) in the identified region.

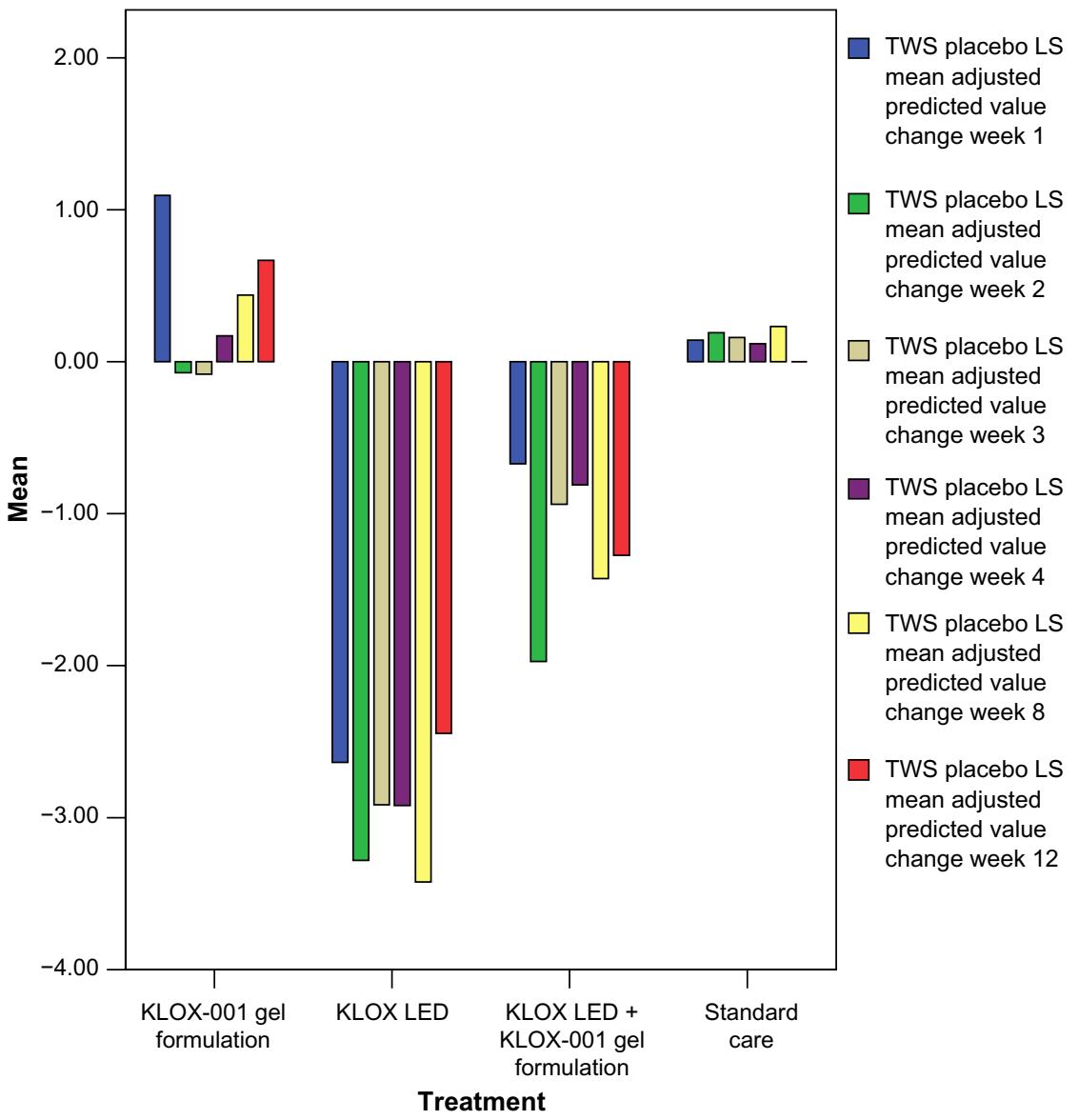

Figure 5 Graphical representation of TWS placebo least-square mean adjusted predicted value change. Abbreviations: LED, light-emitting diode; TWS, total wrinkle score; LS, least-square. 


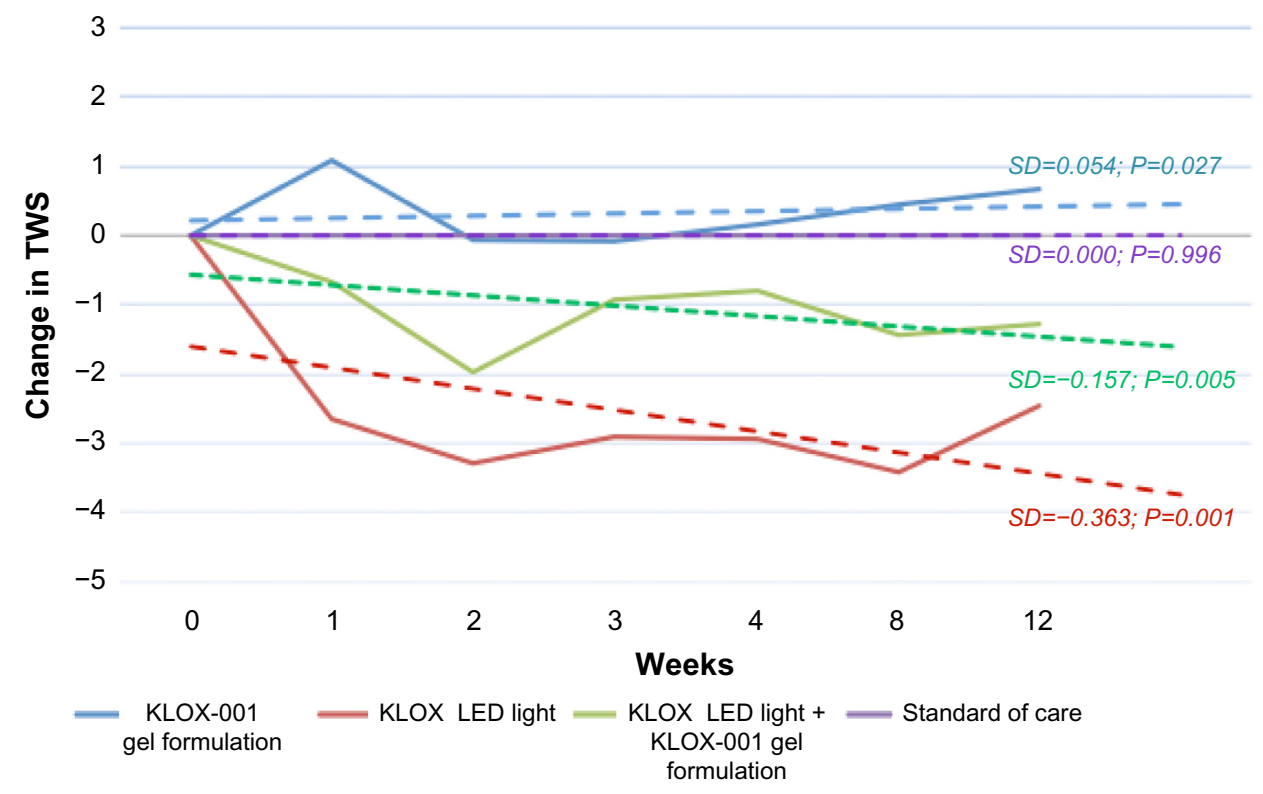

Figure 6 TWS placebo least-square mean adjusted predicted value change.

Abbreviations: LED, light-emitting diode; TWS, total wrinkle score; SD, standard deviation.

Table 3 Physicians' assessment results

\begin{tabular}{|c|c|c|c|c|c|}
\hline & $\begin{array}{l}\text { Group A } \\
\text { KLOX-00I gel } \\
\text { formulation }\end{array}$ & $\begin{array}{l}\text { Group B } \\
\text { KLOX LED } \\
\text { light }\end{array}$ & $\begin{array}{l}\text { Group C } \\
\text { KLOX-00I gel } \\
\text { formulation }+ \\
\text { KLOX LED light }\end{array}$ & $\begin{array}{l}\text { Group D } \\
\text { Standard } \\
\text { treatment }\end{array}$ & P-value \\
\hline & $\mathbf{n}$ & $\mathbf{n}$ & $\mathbf{n}$ & $\mathbf{n}$ & \\
\hline \multicolumn{6}{|l|}{ Brow position } \\
\hline Treatment favored & - & 1 & 5 & - & \\
\hline No difference & 24 & 23 & 19 & 18 & 0.001 \\
\hline \multicolumn{6}{|l|}{ Perioral wrinkling } \\
\hline Treatment favored & - & - & 2 & - & \\
\hline No difference & 24 & 24 & 22 & 18 & 0.001 \\
\hline
\end{tabular}

Table 4 Skin biopsy results (collagen in Gomori Trichrome staining)

\begin{tabular}{|c|c|c|c|c|c|c|c|c|c|c|c|}
\hline \multicolumn{3}{|c|}{$\begin{array}{l}\text { Group A } \\
\text { KLOX-00I gel formulation + } \\
\text { sham light }\end{array}$} & \multicolumn{3}{|c|}{$\begin{array}{l}\text { Group B } \\
\text { Sham gel + KLOX LED light }\end{array}$} & \multicolumn{3}{|c|}{$\begin{array}{l}\text { Group C } \\
\text { KLOX-00 I gel formulation + } \\
\text { KLOX LED light }\end{array}$} & \multicolumn{3}{|c|}{$\begin{array}{l}\text { Group D } \\
\text { Standard treatment }\end{array}$} \\
\hline ID \# & Week 0 & Week 12 & ID \# & Week 0 & Week 12 & ID \# & Week 0 & Week I2 & ID \# & Week 0 & Week I2 \\
\hline 100 & 0.5 & 2 & 106 & 0 & 0 & 102 & 0 & 3 & 103 & I & 0 \\
\hline 101 & 1 & 2 & 107 & 0.5 & 2 & 104 & 0 & I & 108 & 0.5 & 3 \\
\hline 105 & 0.5 & 3 & 110 & 1 & 2.5 & 114 & 0.5 & 1.5 & 118 & 0.5 & 3 \\
\hline 109 & 0 & 2 & 112 & 1 & 2.5 & 116 & 0.5 & 1.5 & 120 & 2 & 2.5 \\
\hline 111 & 0.5 & I & 122 & 0.5 & 3 & 121 & 1 & 0 & 125 & 0.5 & 3 \\
\hline 115 & I & 3 & 123 & 0.5 & 1.5 & 124 & 0 & 3 & $13 \mid$ & 0 & 1.5 \\
\hline 117 & 0.5 & 1.5 & 126 & 0.5 & 3 & 127 & I & 3 & & & \\
\hline 128 & 3 & 3 & 129 & 0.5 & 3 & 130 & 0 & 2 & & & \\
\hline Av. & 0.875 & 2.188 & Av. & 0.563 & 2.188 & Av. & 0.375 & 1.875 & Av. & 0.750 & 2.167 \\
\hline \multicolumn{2}{|c|}{ Change from week 0} & $+150 \%$ & \multicolumn{2}{|c|}{ Change from week 0} & $+287 \%$ & \multicolumn{2}{|c|}{ Change from week 0} & $+400 \%$ & \multicolumn{2}{|c|}{ Change from week 0} & $+189 \%$ \\
\hline
\end{tabular}

Note: Mean score at week 0 (baseline) and at week 12 for each treatment group was calculated, allowing the determination of the percentage of change from week 0. Abbreviation: LED, light-emitting diode; ID, identification; Av., average. 

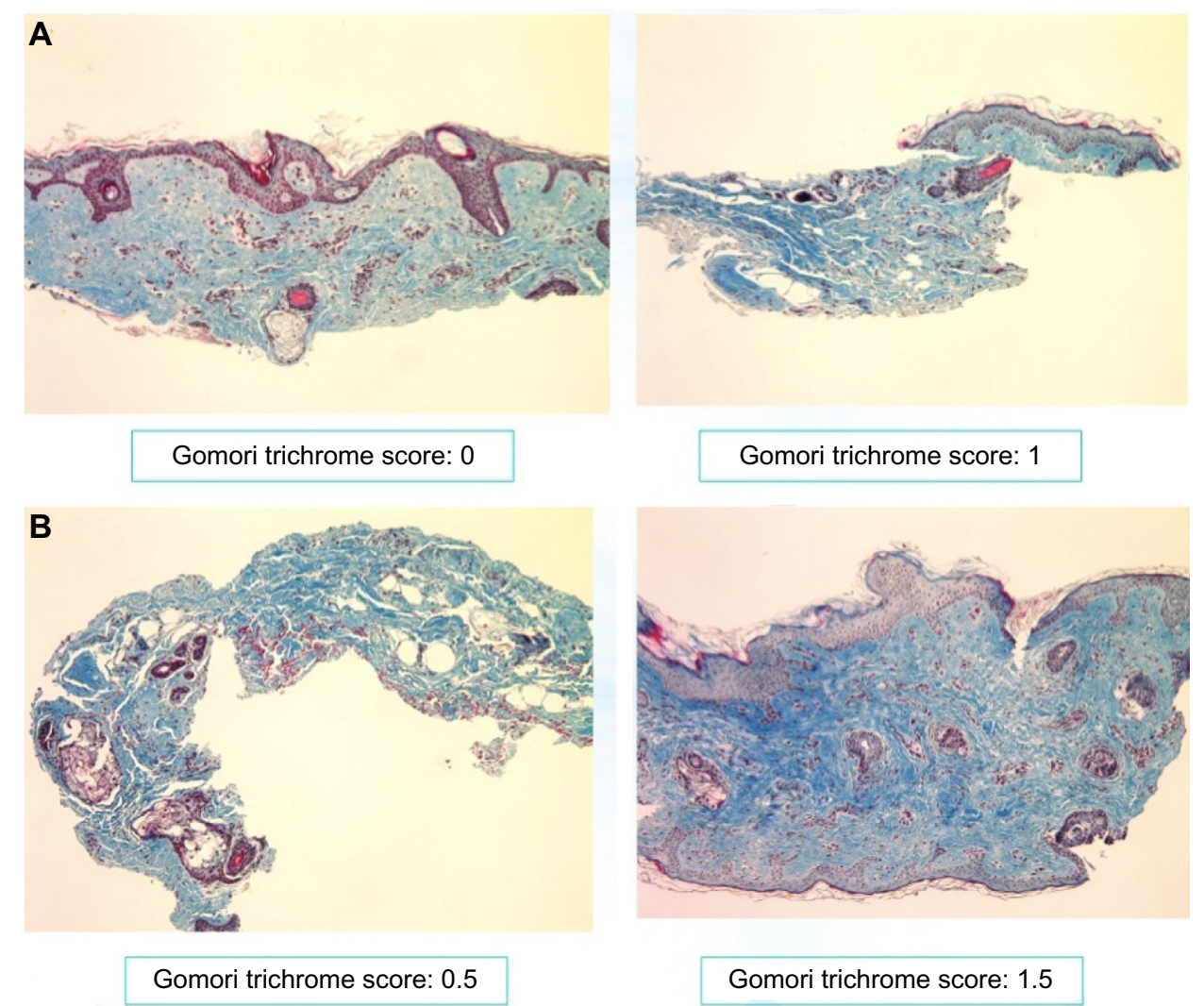

Figure 7 Gomori staining of retroauricular biopsy samples: (A) week 0; (B) week 12.

Notes: A visual increase of the amount of collagen (colored in blue with Gomori trichrome) was still visible 12 weeks after the first treatment, with apparition of dense and thick fascicles in the papillary dermis.

This study evaluated a biophotonic model of skin rejuvenation through a randomized and placebo-controlled clinical trial. Clinical outcome measures were assessed through compilation of various wrinkle severity and facial esthetic grading scales. The use of multiple scales with various weight values on the subjective assessment of skin appearance in a small sample number of patients led to challenges in data analysis. The decision to consolidate multiple outcome measures into a TWS for data analysis was made in order to compensate for discrepancies and variability in clinical scoring, as well as to allow ease of analysis and interpretation of results.

Analysis of both subjective physician assessment and TWS least-square mean adjusted predicted value changes demonstrated that the groups treated with KLOX LED light with KLOX placebo/base gel and KLOX LED light + KLOX-001 gel formulation had superior performance when compared to the other treatment groups, with statistically

Table 5 Summary of treatment-related adverse events

\begin{tabular}{|c|c|c|c|c|c|}
\hline & Group A & Group B & Group C & Group D & \\
\hline & $\begin{array}{l}\text { KLOX-00I gel } \\
\text { formulation }\end{array}$ & $\begin{array}{l}\text { KLOX LED } \\
\text { light }\end{array}$ & $\begin{array}{l}\text { KLOX-00I gel } \\
\text { formulation + } \\
\text { KLOX LED light }\end{array}$ & $\begin{array}{l}\text { Standard } \\
\text { treatment }\end{array}$ & subjects \\
\hline & $n=8$ & $n=8$ & $n=8$ & $n=8$ & $n=32$ \\
\hline & n (\%) & n (\%) & n (\%) & n (\%) & n (\%) \\
\hline Total numbers and & $5(63)$ & $2(25)$ & $6(75)$ & $4(50)$ & $17(53)$ \\
\hline \multicolumn{6}{|l|}{ percentages of subjects } \\
\hline \multicolumn{6}{|l|}{ with TEAEs } \\
\hline Erythema & $3(38)$ & I (I3) & $0(0)$ & $2(25)$ & $6(19)$ \\
\hline Brow edema & I (I3) & I (I3) & $2(25)$ & $0(0)$ & $4(13)$ \\
\hline Eye lid edema & $2(25)$ & $0(0)$ & I (I3) & $0(0)$ & $3(9)$ \\
\hline
\end{tabular}

Abbreviation: LED, light-emitting diode; TEAEs, treatment emergent adverse events. 


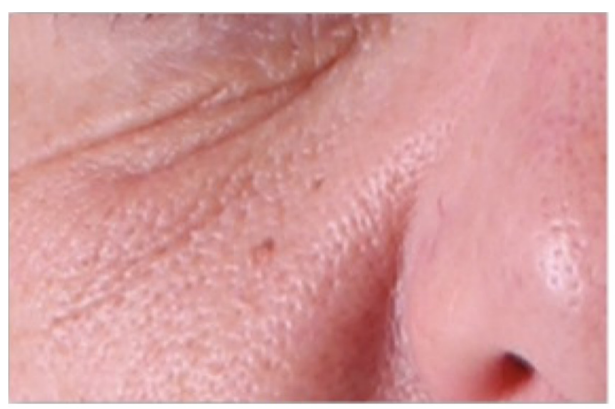

Week 0

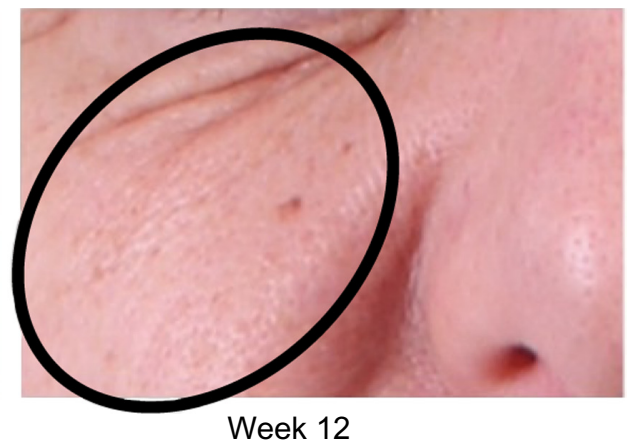

Figure 8 Pore size improvement at week 12

significant results obtained for subjective brow position and TWS.

The patients receiving SOC treatment showed significant deterioration in patient assessment and perception during the course of the study. This necessitated placebo and predicted value adjustments to be made for analysis, as a positive therapeutic effect would have constituted less deterioration relative to the standard group.

The use of a large number of scales with variable weight values on the subjective assessment of skin appearance is an important problem that confounds the study results and may prevent the demonstration of benefits of the combined treatment with KLOX gel and KLOX LED light. Although the KLOX LED light + KLOX-001 gel formulation group demonstrated superior performance in the subjective clinical assessment wing of the study, TWS analysis favored the KLOX LED light with KLOX placebo/base gel group. This may be due to the analysis of TWS as an amalgamation of various differently weighted scales, as well as the small patient population. A larger study may be able to further elucidate the actual discrepancies between the treatments; nevertheless, both the KLOX LED light with KLOX placebo/base gel and KLOX LED light + KLOX001 gel formulation groups fared better than the other two groups.

The rate of collagen deposition was increased in a substantial fashion with KLOX-001 gel formulation + the KLOX LED light compared to other treatment groups.

KLOX-001 gel formulation and KLOX LED light, used alone or in combination, were found to be safe and well tolerated. All AEs reported in each treatment group were mild in intensity, without any subject being discontinued from the study due to AE. Brow edema, which did occur in $13 \%$ of patients, did not affect the patients' overall appearance, was considered mild, and may, in fact, have contributed to the improved skin appearance in the region.
Histopathological results did not demonstrate any safety concern with the use of KLOX topical formulation and KLOX LED light, when used alone or in combination.

Finally, an extensive list of factors was evaluated by a blinded committee, and direct physician evaluation, using standardized and validated questionnaires as well as tissue biopsies and direct patient feedback demonstrating several outcomes repeatedly being reported following treatment.

Several patients commented on a subjective "tightening" of their skin, improvement in pore size, skin texture, and overall appearance (Figure 8).

The use of specific KLOX chromophores has been demonstrated to specifically up- or downregulate cellular mechanisms that impact the normal healing cascade. The use of blue light itself has been shown to affect signaling mechanisms (eg, fibroblast growth factor), while natural cutaneous chromophores may have a bigger impact than what was previously understood, when combined with light that supports an appropriate wavelength or energy transfer. The true role of a chromophore-based rejuvenation strategy is in the combination of up- and downregulating signals leading to a favorable rejuvenation outcome.

Although increased collagen deposition is of principal and paramount importance in any rejuvenation regimen, it does not account for all aspects of the results demonstrated in the present study. Future directions will require the following: an evaluation of the moisture/hydration effect of the technology using capacitance tools, photometric evaluation of skin sebum production, measurement of skin $\mathrm{pH}$, and measurement of skin's viscoelastic properties following treatment with the current biophotonic platform.

\section{Conclusion}

The study results demonstrate that KLOX LED light with placebo/base gel and KLOX-001 gel formulation combined with KLOX LED light are effective, safe, well-tolerated, and 
painless treatment modalities for skin rejuvenation. They avoid the inherent disadvantages and risks of ablative and nonablative thermal techniques, thereby decreasing costs, patient discomfort, and downtime. Future directions will further analyze the direct benefits of hydration and sebum production.

\section{Disclosure}

Andreas Nikolis and Steven Bernstein are consultants with KLOX Technologies. Shipra Rastogi was an employee of KLOX Technologies. The authors report no other conflicts of interest in this work.

\section{References}

1. Doherty SD, Doherty CB, Markus JS, Markus RF. A paradigm for facial skin rejuvenation. Facial Plast Surg. 2009;25:245-251.

2. Rabe JH, Mamelak AJ, McElgunn PJ, Morison WL, Sauder DN. Photoaging: mechanisms and repair. J Am Acad Dermatol. 2006;55:1-19.

3. Gilchrest BA. Skin aging and photoaging: an overview. J Am Acad Dermatol. 1989;21:610-613.

4. Uitto J, Bernstein EF. Molecular mechanisms of cutaneous aging: connective tissue alterations in the dermis. J Investig Dermatol Symp Proc. 1998;3:41-44.

5. Braveman IM, Fonferko E. Studies in cutaneous aging, the elastic fiber network. J Invest Dermatol. 1982;78:434-443.

6. Montagna W, Kirchner S, Carlisle K. Histology of sundamaged human skin. J Am Acad Dermatol. 1989;21:907-918.

7. El-Domyati M, Attia S, Saleh F, et al. Intrinsic aging vs. photoaging: a comparative histopathological, immunohistochemical, and ultrastructural study of skin. Exp Dermatol. 2002;11:398-405.

8. Kondo S. The roles of cytokines in photoaging. J Dermatol Sci. 2000;23:30-36.

9. Lee SY, Park K-H, Choi J-W, et al. A prospective, randomized, placebocontrolled, double-blinded, and split-faced clinical study on LED phototherapy for skin rejuvenation: Clinical, profilometric, histologic, ultrastructural, and biochemical evaluations and comparison of three different treatment settings. J Photochem Photobiol. B 2007;88:51-67.

10. Branham GH, Thomas JR. Rejuvenation of the skin surface: chemical peel and dermabrasion. Facial Plast Surg. 1996;12:125-133.

11. Arian LE, Hruza GJ. Current lasers in skin resurfacing. Facial Plast Surg Clin North Am. 2005;13:127-139.

12. Orringer JS, Kang S, Johnson TM, et al. Connective tissue remodeling induced by carbon dioxide laser resurfacing of photodamaged human skin. Arch Dermatol. 2004;140:1326-1332.

13. Weiss RA, McDaniel DH, Geronemus RG. Review of nonablative photorejuvenation: Reversal of the aging effects of the sun and environmental damage using laser and light sources. Semin Cutan Med Surg. 2003;22:93-106.

14. Butler PE, Gonzales S, Randolph MA, Kim J, Kollias N, Yaremchuk MJ. Quantitative and qualitative effects of chemical peeling on photoaged skin: an experimental study. Plast Reconstr Surg. 2001;107: 222-228.

15. Dover JS, Hruza G. Lasers in skin resurfacing. Australas J Dermatol. 2000;41:72-85.

16. Nanni CA, Alster TS. Complications of carbon dioxide laser resurfacing: an evaluation of 500 patients. Dermatol Surg. 1998;24:315-320.

17. Spiprachya-Anunt S, Fitzpatrick RE, Goldman MP, Smith SR. Infections complicating pulsed carbon dioxide laser resurfacing for photoaged skin. Dermatol Surg. 1997;23:527-535.
18. Hardaway CA, Ross EV. Nonablative laser skin remodeling. Dermatol Clin. 2002;20(1):97-111.

19. Dierickx CC, Anderson RR. Visible light treatment of photoaging. Dermatol Ther. 2005;18:191-208.

20. Weiss RA, Weiss MA, Geronemus RG, McDaniel DH. A novel nonthermal non-ablative full panel LED photomodulation device for reversal of photoaging: digital microscopic and clinical results in various skin types. J Drugs Dermatol. 2004;3:605-610.

21. Nelson JS, Majaron B, Kelly KM. What is nonablative photorejuvenation of human skin? Semin Cutan Med Surg. 2002;21:238-250.

22. Sadick NS. Update on nonablative light therapy for rejuvenation: a review. Lasers Surg Med. 2003;32:120-128.

23. Sommer AP, Pinheiro AP, MEster AR, Franke RP, Whelan HT. Biostimulatory windows in low-intensity laser activation: lasers, scanners and NASA's light-emitting diode array system. J Clin Laser Med Surg. 2001;19:29-33.

24. Whelan HT, Buchmann EV, Dhokalia A, et al. Effect of NASA lightemitting diode on molecular changes for wound healing in diabetic mice. J Clin Laser Med Surg. 2003;21:67-74.

25. Whelan HT, Connelly JF, Hodgson BD, et al. NASA light-emitting diode for the prevention of oral mucositis in pediatric bone marrow transplant patients. J Clin Laser Med Surg. 2002;20:319-324.

26. Weiss RA, McDaniel DH, Geronemus R, Weiss MA. Clinical trial of a novel non-thermal LED array for the reversal of photoaging: clinical, histologic, and surface profilometric results. Lasers Surg Med. 2005;36:85-91.

27. Barolet D, Roberge CJ, Auger FA, Boucher A, Germain L. Regulation of skin collagen metabolism in vitro using a pulsed $660 \mathrm{~nm}$ LED light source: clinical correlation with a single-blinded study. J Invest Dermatol. 2009;129:2751-2759.

28. Russell BA, Kellett N, Reilly LR. A study to determine the efficacy of combination LED light therapy (633 and $830 \mathrm{~nm}$ ) in facial skin rejuvenation. J Cosmet Laser Ther. 2005;7:196-200.

29. Goldberg DJ, Amin S, Russell BA, Phelps R, Kellett N, Reilly LA. Combined $633 \mathrm{~nm}$ and $830 \mathrm{~nm}$ LED treatment of photoaging skin. J Drugs Dermatol. 2006;5:748-753.

30. Nikolis A, Morissette C, Rastagi S, Loepis N, Piergallini R, Hebert L. Use of chromophores in wound healing. In: 2009 Canadian Association of Wound Care; October 29; 2009; Quebec City, Canada. KLOX Technologies, data on file.

31. Honeck P, Weiss C, Sterry W, Rzany B; Gladys study group. Reproducibility of a four-point clinical severity score for glabellar frown lines. Br J Dermatol. 2003;149:306-310.

32. Fitzpatrick RE, Goldman MP, Satur NM, Tope WD. Pulsed carbon dioxide laser resurfacing of photo-aged facial skin. Arch Dermatol. 1996;132:395-402.

33. Carruthers A, Carruthers J, Hardas B, et al. A validated grading scale for marionette lines. Dermatol Surg. 2008;34(Suppl 2):S167-S172.

34. Carruthers A, Carruthers J, Hardas B, et al. A validated lip fullness grading scale. Dermatol Surg. 2008;34(Suppl 2):S161-S166.

35. Shoshani D, Markovitz E, Monstrey SJ, Narins DJ. The modified Fitzpatrick Wrinkle Scale: a clinical validated measurement tool for nasolabial wrinkle severity assessment. Dermatol Surg. 2008;34(Suppl 1): S85-S91.

36. Carruthers A, Carruthers J, Hardas B, et al. A validated grading scale for forehead lines. Dermatol Surg. 2008;34(Suppl 2):S155-S160.

37. Carruthers A, Carruthers J, Hardas B, et al. A validated grading scale for crow's feet. Dermatol Surg. 2008;34(Suppl 2):S173-S178.

38. Lemperle G, Holmes RE, Cohen SR, Lemperle SM. A classification of facial wrinkles. Plast Reconstr Surg. 2001;108:1735-1750.

39. Day DJ, Littler CM, Swift RW, Gottlieb S. The wrinkle severity rating scale: a validation study. Am J Clin Dermatol. 2004;5:49-52.

40. Glogau RG. Aesthetic and anatomic analysis of the aging skin. Semin Cutan Med Surg. 1996;15:134-138. 


\section{Publish your work in this journal}

Clinical, Cosmetic and Investigational Dermatology is an international, peer-reviewed, open access, online journal that focuses on the latest clinical and experimental research in all aspects of skin disease and cosmetic interventions. All areas of dermatology will be covered; contributions will be welcomed from all clinicians and basic science researchers globally. This journal is indexed on CAS. The manuscript management system is completely online and includes a very quick and fair peer-review system, which is all easy to use. Visit http://www.dovepress.com/testimonials.php to read real quotes from published authors.

Submit your manuscript here: http://www.dovepress.com/clinical-cosmetic-and-investigational-dermatology-journal 